\title{
A Method for the Analysis of Interference from DME to ATCRBS in the Time Domain
}

\author{
Guofeng Jiang ${ }^{1,2, * \mathbb{D}}$, Yangyu Fan ${ }^{1}$, Hongbo Yuan ${ }^{2}$ and Pengliang Yuan ${ }^{1}$ \\ 1 School of Electronics and Information, Northwestern Polytechnical University, Xi'an 710072, China; \\ fan_yangyu@nwpu.edu.cn (Y.F.); hamsir@126.com (P.Y.) \\ 2 Aviation Maintenance School for NCO, Air Force Engineering University, Xinyang 464000, China; \\ hongbo_yuan76@163.com \\ * Correspondence: jgf_6921@mail.nwpu.edu.cn
}

Received: 5 March 2019; Accepted: 28 March 2019; Published: 1 April 2019

check for updates

\begin{abstract}
Analysis of the coexistence of two or more types of equipment is increasingly important. However, at present studies on the analysis method in the time domain are scant. Therefore, the aim of this paper is to explore the characteristics of signals and relations between interfering and desired signals in the time domain. Based on the periodicity of a signal, this paper presents a Periodic Pulse Overlap Method (PPOM). Using PPOM to analyze the interference from Distance Measuring Equipment (DME) to Air Traffic Control Radar Beacon System (ATCRBS) in the time domain, we obtain almost the same result as that based on the Monte Carlo Method (MCM). Furthermore, we discover the measures to reduce or even avoid interference, such as changing the Pulse Recurrence Frequency (PRF), adjusting the difference of initial time, and switching the operating modes of the equipment.
\end{abstract}

Keywords: coexistence; DME; ATCRBS; PPOM

\section{Introduction}

With the increase of electrical and electronic equipment in recent years, analysis of the coexistence between them has gathered great importance. Interference is often analyzed in the frequency domain, power domain, and signal processing, and some researchers have analyzed coexistence between two pieces of equipment operating at close frequency [1-3]. Takuya and Shigeru [4] propose an estimation method for the signal environment in ARNS (aeronautical Radio Navigation Service: 960 1215 MHz) band to examine whether equipment sharing a frequency band interfere with each other. However, the analysis of coexistence in the frequency or power domain provides the worst case results because the involved systems are assumed to transmit continuously [5], the result is consistent with the actual value only when baseband signal is analog, but the result is different from the actual value when the signal is pulsed with small duty cycle, since the interfering and desired signal do not overlap with each other most of the time, i.e., interference does not occur all the time. Some researchers use signal processing to analyze the coexistence between two pieces of equipment, for example Khodr A et al. [6] studied the cancellation of DME interference for aeronautical communications using signal processing. Miguel A. [7] assessed the impact of L-band digital aeronautical communications system (LDACS) on JTIDS (a military radio system known as Joint Tactical Information Distribution System) using signal processing based on some assumptions for JTIDS. Nevertheless, there are some limitations when using signal processing to analyze interference, since the detailed characteristics of signals are not open, especially for military equipment (e.g., JTIDS). 
Both DME and ATCRBS are types of aeronautical radio equipment, and operate in the L-band at the same time, so coexistence between the two pieces of equipment must be analyzed to ensure that they can work properly. To avoid the interference between DME and ATCRBS when they are located in the same airplane, they operate in time division multiplexing access (TDMA). However, it is difficult for using TDMA if pieces of equipment are located in the different airplanes, and if two airplanes are near enough, interfering power from an airplane can impact on sensitive equipment fitted on the other airplane, hence it is important to analyze interference in the time domain to evaluate the performance degradation of the interfered equipment and discover the reason for interference. Najett et al. [8] have verified the impact of the interference using average capacity based on the Shannon-Hartley theorem through analyzing the influence of channel occupation, and thought that time-domain approach seems to be more accurate. Generally, the analysis of coexistence in the time domain can be achieved by calculating the overlap probability between the interfering and desired signals.

There are two methods on analyzing the overlap between two or more pulse signals; one is known as Poisson Distribution Method (PDM), and the other is called Monte Carlo Method (MCM). The assumption is adopted that the pulses are randomly distributed with respect to the time forming Poisson process in PDM. Vassilios [3] explained the mechanisms of JTIDS interference on DME in the time domain based on PDM. However, the interfering and desired signals are periodic in most cases, the pulse-stream does not correspond with the Poisson distribution if the number of signals is not large enough. The pulse-stream can be thought to be corresponding with the Poisson distribution only when there are multi-path periodic signals, and then PDM can be used to analyze the interference. Najett et al. [9] have analyzed the interference of LDACS on DME in the time domain based on a Monte Carlo process, and obtained the result that their coexistence is possible, however, It takes a lot of time for MCM to analyze interference to ensure the accuracy. Many researchers have studied interference between two or more periodic pulse signals using the mean probability of pulse collision [10,11], but they have not studied the impact of PRF and initial time on the probability of pulse collision. This paper presents a PPOM to calculate the overlap probability between two or more periodic pulse signals. Moreover, we study the parameters affecting overlap probability such as PRF, pulse duration, and the difference of initial time between interfering and desired signals. Compared with PDM, PPOM can be used to analyze single-path or multi-path interference source. PPOM is different from MCM, since PPOM is an analytic method and needs less time to analyze.

In this paper, we apply PPOM to analyze the interference of DME on ATCRBS in the time domain, and then provide corresponding measures to reduce or avoid interference impact from DME. The remainder of the paper is organized as follows. In Section 2, we revisit the principles of DME and ATCRBS briefly, and focus on the technical characteristics of the two systems that are pertinent to the analysis of interference such as signal waveform, pulse duration, and PRF. In Section 3, we build a model to analyze the interference of DME on ATCRBS, expound and prove the periodicity of pulse overlap, and study the characteristics of pulse overlap. In Section 4, we use PDM and MCM and PPOM to analyze the interference of DME on ATCRBS, respectively, and then simulate the variance of recognition probability with respect to difference of initial time or multiples of PRF between interfering and desired signals. Finally, we provide conclusions in Section 5.

\section{Overview of DME and ATCRBS}

\subsection{Overview of $D M E$}

DME is an aeronautical radio navigation system in use for decades and operating in $960 \sim 1215 \mathrm{MHz}$ with $1 \mathrm{MHz}$ interval. DME is composed of airborne interrogator and ground transponder. DME provides slant distance between the aircraft and the ground station. The airborne interrogator transmits a pair of pulses named as DME interrogation, when the ground transponder receives the pair of pulses, waits $50 \mu s$, 
and then transmits another pair of pulses named as DME reply to the aircraft, so the airborne transceiver can estimate the range through measuring the time difference between transmission and reception.

DME pulse pair consisted of two pulses with different interval according to the operation mode. The signal waveform of DME pulse pair is depicted in Figure 1, in fact, the pulse is Gaussian shaped, we use rectangular pulse signal to represent the Gaussian for simplicity. $I_{2}$ denotes the spacing of the pulses and depends on the certain mode of the DME station, which is $12 \mu$ s for mode $\mathrm{X}$ interrogation, $30 \mu \mathrm{s}$ for mode $\mathrm{X}$ reply, and $36 \mu$ s for mode $\mathrm{Y}$ interrogation or reply, respectively [12]. $L_{2}$ is the first idle duration and $S_{2}$ is the second idle duration in one cycle. $T_{2}$ denotes the recurrence period of interrogation or reply.

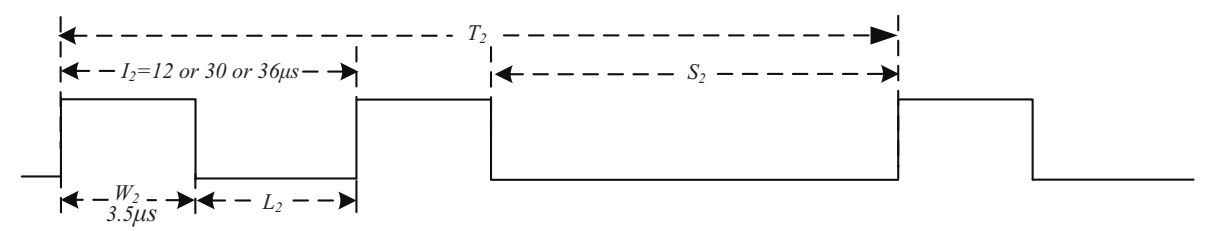

Figure 1. The basic pulse pair of Distance Measuring Equipment (DME) signal.

\subsection{Overview of ATCRBS}

ATCRBS is generally used to be an aid to air traffic services, and consists of the Secondary Surveillance Radar (SSR) and Air Traffic Control (ATC) transponder. SSR transmits interrogation signals using a $1030 \mathrm{MHz}$ carrier to the ATC transponder, when the ATC transponder receives a valid interrogation, and then return a reply signal using a $1090 \mathrm{MHz}$ carrier to the interrogator. If the interrogation mode is $\mathrm{A}$ or $\mathrm{C}$, then ATC transponder replies for identity or automatic pressure-altitude accordingly [13].

The waveform of SSR interrogation signal is depicted in Figure 2. It consists of P1 and P2 and P3 pulse with the same pulse width $\left(W_{1}=0.8 \mu \mathrm{s}\right) . I_{1}$ denotes the spacing between pulse $\mathrm{P} 1$ and $\mathrm{P} 3$, and depends on the certain mode of the interrogation mode, $I_{1}$ is equal to $8.0 \mu$ s for mode $A, 21 \mu$ s for mode $C$ respectively. Pulse P2 is used to suppress any replies to side lobe interrogation, and ATC transponders reply only when the level of P2 is lower than $9 \mathrm{~dB}$ below P1. The interval between P1 and P2 is $2 \mu \mathrm{s}$. The PRF of SSR interrogation signal is $150 \sim 400 \mathrm{~Hz}$.

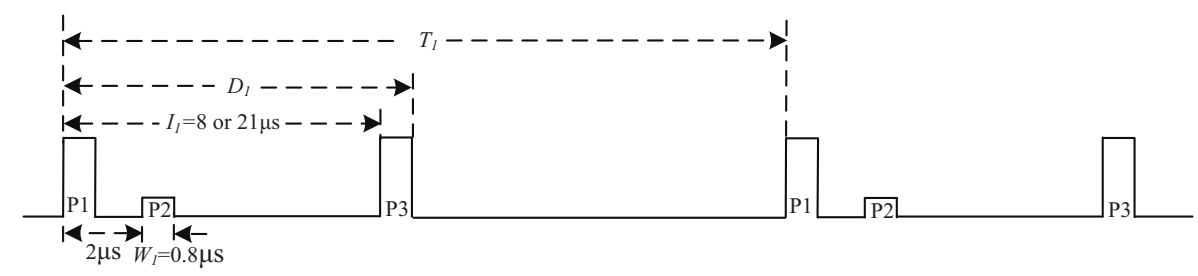

Figure 2. The baseband signal of Secondary Surveillance Radar (SSR) interrogation.

The structure of ATC reply signal is depicted in Figure 3, which consists of framing pulses (F1 and F2), information pulses (A1A2A4, B1B2B4, C1C2C4, and D1D2D4), and special position identification pulse (SPI). Framing pulses spacing $20.3 \mu$ s are the most elementary code and always exist, while SPI follows the last framing pulse of Mode A replies only as a result of manual selection. Information pulses are the codes of identification for mode $A$ or pressure altitude for mode $C$ and the " $X$ " pulse is specified only as a technical standard to safeguard possible future use. PRF of ATC reply signal is 500 2000 Hz. 


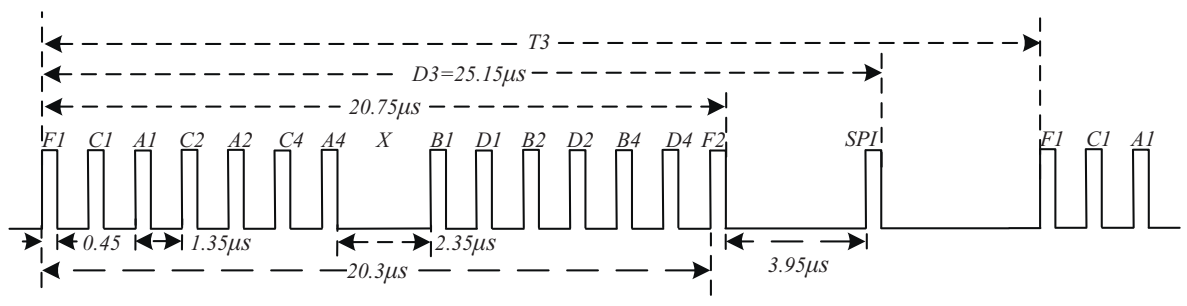

Figure 3. The baseband signal of SSR reply.

\subsection{Reply Efficiency of ATCRBS}

Reply efficiency is the fundamental characteristic of ATCRBS, which equals to the ration of the number of reply signal to that of interrogation. Then reply efficiency of ATCRBS represented by $R_{e}$ can be defined as follows:

$$
R_{e}=\frac{N_{\text {int_rep }}}{N_{\text {int_int }}}=\frac{N_{\text {int_rep }}}{N_{\text {trans_rep }}} \cdot \frac{N_{\text {trans_rep }}}{N_{\text {int_int }}}=P_{\text {inter }} \cdot P_{\text {reply }}
$$

where $N_{\text {int_rep }}$ is the number of reply signals received by SSR, $N_{\text {int_int }}$ is the number of interrogation signals received by ATC, $N_{\text {trans_rep }}$ is the amount of reply signals transmitted by ATC, $P_{\text {int }}=\frac{N_{\text {int_rep }}}{N_{\text {trans_rep }}}$ is the recognition probability of interrogation signal, and $P_{\text {reply }}=\frac{N_{\text {trans_rep }}}{N_{\text {int_int }}}$ is the recognition probability of reply signal. It can be seen that $R_{e}$ is equal to 1 only when all the interrogation and reply signals are recognized. However, the interrogation and reply signals cannot be recognized totally owing to interference in most cases, so $R_{e}$ is lesser than 1 in general.

\section{Analysis and Derivation of PPOM Formula}

PPOM is an analytical method based on the periodicity of pulse signal, the overlap probability between two or more pulse signals is used to characterize the interference probability, so the key to PPOM is the calculation of overlap probability. Analysis and derivation of overlap probability are discussed in this section. Firstly, we build a model of pulse overlap based on the signal structure of DME and ATCRBS; secondly, we expound and prove the periodicity of pulse overlap; thirdly, we derive the formula of the probability of pulse overlap and study the variation of number in a cycle. Finally, we derive the formula of the mean overlap probability for DME and ATCRBS.

\subsection{Modeling of Pulse Overlap for DME and ATCRBS}

Pulse overlap is also known as pulse collision, which is defined as the rising edge time of the back pulse is earlier than the falling edge time of the front pulse in a pulse-stream. If there are two pulses with different duration, moreover, both rising and falling edge times of the narrow pulse are in the range of wide pulse duration, then the narrow pulse is considered to be overlapped by wide pulse fully. In fact, it can be named as overlap if the duration of one event covers that of the other event partly or fully. Overlap occurs not only between two pulses but also between pulse and idle duration.

According to the signal structure of DME and ATCRBS, the model for pulse overlap analysis in the time domain is built and illustrated in Figure 4. There are two periodic pulse signals; one pulse signal is $\mathrm{PL}_{1}$ representing ATCRBS signal, the other pulse signal $\mathrm{PL}_{2}$ composed of two pulses in one cycle represents DME signal. The pulse duration of $\mathrm{PL}_{1}$ is $\tau_{1}$, which denotes $D_{1}$ in Figure 2 or $D_{3}$ in Figure 3. $T_{1}$ and $T_{2}$ represent the period of $\mathrm{PL}_{1}$ and $\mathrm{PL}_{2}$, respectively. The symbol $t_{11}$ and $t_{21}$ represent the initial time and $\Delta t_{0}$ is the difference between $t_{11}$ and $t_{21}$. The definitions of the other symbols in PL 2 are the same as that shown in Figure 1. 


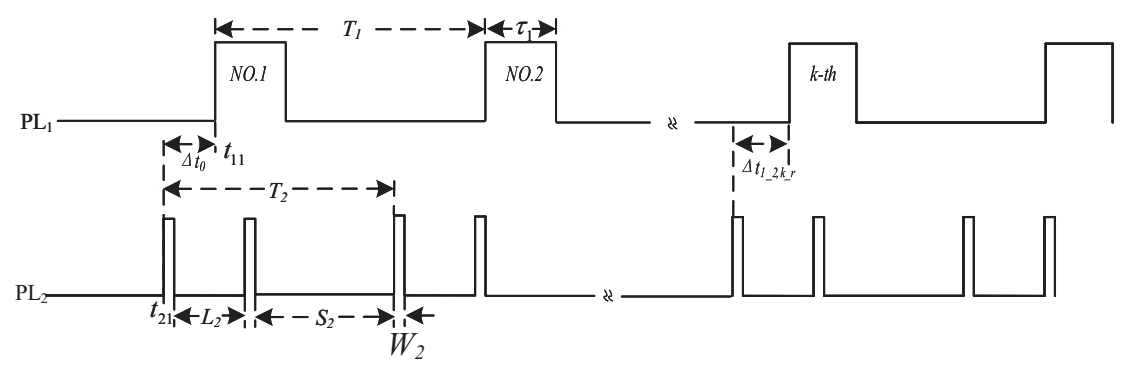

Figure 4. The baseband signal of SSR reply.

There are two idle times in $\mathrm{PL}_{2}$ represented by $L_{2}$ and $S_{2}$. PL $L_{1}$ will not overlap with $\mathrm{PL}_{2}$ if $\tau_{1}$ is overlapped by $L_{2}$ or $S_{2}$ completely. If $t_{1 k}$ denotes the rising edge time of the $k$-th pulse of $\mathrm{PL}_{1}$, the difference represented by $\Delta t_{1_{2}, k_{r}}$ between $t_{1 k}$ and the rising edge time of $\mathrm{PL}_{2}$ located in the front adjacent to the $k-t h$ of $\mathrm{PL}_{1}$ is calculated to be:

$$
\begin{aligned}
& \Delta t_{1 \_2, k \_r}=t_{11}+(k-1) \cdot T_{1}-\left(t_{21}+\left\lfloor\frac{t_{11}+(k-1) \cdot T_{1}-t_{21}}{T_{2}}\right\rfloor \cdot T_{2}\right) \\
& =\Delta t_{0}+(k-1) \cdot T_{1}-\left\lfloor\frac{\Delta t_{0}+(k-1) \cdot T_{1}}{T_{2}}\right\rfloor \cdot T_{2} \\
& =\left[(k-1) \cdot T_{1}+\Delta t_{0}\right] \bmod T_{2}
\end{aligned}
$$

where $\left[(k-1) \cdot T_{1}+\Delta t_{0}\right] \bmod T_{2}$ is the remainder from dividing $(k-1) \cdot T_{1}+\Delta t_{0}$ by $T_{2} . L_{2}$ will overlap with the $k$-th pulse of $\mathrm{PL}_{1}$ fully if the following inequality condition is satisfied:

$$
0<\Delta t_{1 \_2, k \_r}-W_{2}<L_{2}-\tau_{1}
$$

Similarly, $S_{2}$ will overlap with the $k-t h$ pulse of $\mathrm{PL}_{1}$ fully if the following inequality condition is satisfied:

$$
0<\Delta t_{1 \_2, k \_r}-L_{2}-2 W_{2}<S_{2}-\tau_{1}
$$

If the $k$-th pulse of $\mathrm{PL}_{1}$ overlaps with $L_{2}$ or $S_{2}$ fully, then overlap probability can be considered to be probability of $\mathrm{PL}_{1}$ pulse non-overlapped by $\mathrm{PL}_{2}$ pulse.

\subsection{Periodicity of Pulse Overlap}

\subsubsection{Calculation for the Period of Pulse Overlap}

Since $\mathrm{PL}_{1}$ and $\mathrm{PL}_{2}$ are periodicity signals, the overlap between $\mathrm{PL}_{1}$ and $\mathrm{PL}_{2}$ should have a cyclical characteristic, and the period of pulse overlap $T_{o}$ can be calculated as follows:

$$
T_{o}=\frac{T_{1} \cdot T_{2}}{g}
$$

where $g$ is the greatest common divisor of $T_{1}$ and $T_{2} \cdot g$ is equal to the ration of the greatest common divisor of numerators to the least common multiple of denominators ratio when $T_{1}$ and $T_{2}$ are fractions. Clearly, the number $N_{T_{1}}$ of pulse $\mathrm{PL}_{1}$ in one overlap cycle can be expressed as follows:

$$
N_{T_{1}}=\frac{T_{2}}{g}
$$


Let the data set $S_{r}\left(\Delta t_{0}\right)$ denote the difference of the rising edge time between $\mathrm{PL}_{1}$ and the first pulse of $\mathrm{PL}_{2}$ in one overlap cycle:

$$
S_{r}\left(\Delta t_{0}\right)=\left\{\Delta t_{0} \bmod T_{2}, \cdots,\left[(k-1) \cdot T_{1}+\Delta t_{0}\right] \bmod T_{2}, \cdots,\left(N_{T_{1}}-1\right) \cdot T_{1} \bmod T_{2}\right\}
$$

3.2.2. Calculation for the Period of $S_{r}\left(\Delta t_{0}\right)$ When $T_{1} \bmod T_{2}=0$

If $T_{1} \bmod T_{2}=0$, the congruence equation is true. $S_{r}\left(\Delta t_{0}\right)$ can be obtained as follows:

$$
S_{r}\left(\Delta t_{0}\right)=\left\{\Delta t_{0} \bmod T_{2}\right\}
$$

So, the period of $\Delta t_{0}$ is $T_{2}$. When $\Delta t_{0}$ varies from 0 to $T_{2}$, all the difference of rising edge time in one overlap cycle are equal. That is to say, the difference of rising edge time has nothing to do with the sequence number $k$, so it must be either all or none of the $\mathrm{PL}_{1}$ pulses overlap with $\mathrm{PL}_{2}$.

3.2.3. Calculation for the Period of $S_{r}\left(\Delta t_{0}\right)$ When $T_{1} \bmod T_{2} \neq 0$

Let natural number set $m \in\left[0, N_{T_{1}}-1\right], N_{T_{1}}$ is defined as Equation (6), given any nature number $m_{n} \in m$, we can solve the congruence equation:

$$
(k-1) \cdot T_{1}+m \cdot g \equiv 0 \bmod \left(T_{2}\right) .
$$

where $k$ is unknown number. Since $m_{n} \cdot g \bmod g=0$ which satisfies the necessary and sufficient conditions for solution to congruence equation, there must exist solutions to satisfy Equation (9), if $k_{0}$ denotes one of its solutions, then the general solution can be expressed as follows [14]:

$$
k=1+k_{0}+N_{T_{1}} \cdot j, j=0, \pm 1, \pm 2 \cdots,
$$

There exists a unique solution designated $k_{n} \in\left[1, N_{T_{1}}\right]$ in the integer set $k$. So we can conduct that:

$$
\left[\left(k_{n}-1\right) \cdot T_{1}+m_{n} \cdot g\right] \bmod T_{2}=0 .
$$

Therefore, the data set $S_{r}\left(\Delta t_{0}\right)$ can be expressed as follows:

$$
\begin{aligned}
& S_{r}\left(\Delta t_{0}\right)=\left\{\Delta t_{0} \bmod T_{2}, \cdots,\left[(k-1) \cdot T_{1}+\Delta t_{0}\right] \bmod T_{2}, \cdots,\left[\left(N_{T_{1}}-1\right) \cdot T_{1}+\Delta t_{0}\right] \bmod T_{2}\right\} \\
& =\left\{\Delta t_{0} \bmod T_{2}, \cdots,\left[\left(k_{n}-1\right) \cdot T_{1}-m_{n} \cdot g+\Delta t_{0}+m_{n} \cdot g\right] \bmod T_{2}, \cdots,\left[\left(N_{T_{1}}-1\right) \cdot T_{1}+\Delta t_{0}\right] \bmod T_{2}\right\} \\
& =\left\{\Delta t_{0} \bmod T_{2}, \cdots,\left(\Delta t_{0}+m_{n} \cdot g\right) \bmod T_{2}, \cdots,\left(\Delta t_{0}+m_{N_{T_{1}}-1} \cdot g\right) \bmod T_{2}\right\}
\end{aligned}
$$

where $m_{1}, m_{2}, \cdots m_{N_{T_{1}}-1} \in\left[1, N_{T_{1}}-1\right]$, moreover, they are different integers. For any $N \in\left\{0,1,2, \cdots, N_{T_{1}}-1\right\}$, we can get:

$$
\begin{aligned}
& S_{r}\left(\Delta t_{0}+N \cdot g\right)=\left\{\left(\Delta t_{0}+N \cdot g\right) \bmod T_{2},\left[\Delta t_{0}+(N+1) g\right] \bmod T_{2} \cdots\left[\Delta t_{0}+\left(N_{T_{1}}-1\right) \cdot g\right] \bmod T_{2}\right. \\
& \left.\left[\Delta t_{0}+\left(N_{T_{1}}-1+1\right) \cdot g\right] \bmod T_{2}, \cdots,\left[\Delta t_{0}+\left(N_{T_{1}}-1+N\right) \cdot g\right] \bmod T_{2}\right\}
\end{aligned}
$$

Note that $\Delta t_{0}+m_{n} \cdot g \equiv \Delta t_{0}+\left(m_{n}+N_{T_{1}}\right) \cdot g\left(\bmod T_{2}\right)$, then $S_{r}\left(\Delta t_{0}\right)$ can be expressed as follows:

$$
\begin{aligned}
& S_{r}\left(\Delta t_{0}+N \cdot g\right)=\left\{\left(\Delta t_{0}+N \cdot g\right) \bmod T_{2},\left[\Delta t_{0}+(N+1) g\right] \bmod T_{2}, \cdots,\left[\Delta t_{0}+\left(N_{T_{1}}-1\right) \cdot g\right] \bmod T_{2},\right. \\
& \left.\left[\Delta t_{0}+\left(N_{T_{1}}-1+1-N_{T_{1}}\right) \cdot g\right] \bmod T_{2}, \cdots,\left[\Delta t_{0}+\left(N_{T_{1}}-1+N-N_{T_{1}}\right) \cdot g\right] \bmod T_{2}\right\} \\
& =\left\{\Delta t_{0} \bmod T_{2},\left(\Delta t_{0}+g\right) \bmod T_{2}, \cdots,\left[\Delta t_{0}+\left(N_{T_{1}}-1\right) \cdot g\right] \bmod T_{2}\right\}=S_{r}\left(\Delta t_{0}\right)
\end{aligned}
$$


Hence $g$ is the period of $\Delta t_{0}$. For a nonnegative integer $n_{0}$ and $0 \leq \varepsilon_{0}<g$, we write $\Delta t_{0}=n_{0} \cdot g+\varepsilon_{0}$. According to Equation (10), the equation $S_{r}\left(\Delta t_{0}\right)=S_{r}\left(\Delta t_{0}-n_{0} \cdot g\right)$ is true, so $S_{r}\left(\Delta t_{0}\right)$ can be expressed as follows:

$$
\begin{aligned}
& S_{r}\left(\Delta t_{0}\right)=\left\{\Delta t_{0} \bmod T_{2},\left(\Delta t_{0}+g\right) \bmod T_{2}, \cdots,\left[\Delta t_{0}+\left(N_{T_{1}}-1\right) \cdot g\right] \bmod T_{2}\right\} \\
& =\left\{\left(\Delta t_{0}-n_{0} \cdot g\right) \bmod T_{2},\left(\Delta t_{0}-n_{0} \cdot g+g\right) \bmod T_{2}, \cdots,\left[\Delta t_{0}-n_{0} \cdot g+\left(N_{T_{1}}-1\right) \cdot g\right] \bmod T_{2}\right\} \\
& =\left\{\varepsilon_{0} \bmod T_{2},\left(\varepsilon_{0}+g\right) \bmod T_{2}, \cdots,\left[\varepsilon_{0}+\left(N_{T_{1}}-1\right) \cdot g\right] \bmod T_{2}\right\}
\end{aligned}
$$

Note that the maximum value $\varepsilon_{0}+\left(N_{T_{1}}-1\right) \cdot g=\varepsilon_{0}+T_{2}-g<T_{2}$, then:

$$
S_{r}\left(\Delta t_{0}\right)=\left\{\varepsilon_{0}, \varepsilon_{0}+g, \cdots \varepsilon_{0}+(k-1) \cdot g, \cdots, \varepsilon_{0}+\left(N_{T_{1}}-1\right) \cdot g\right\}
$$

\subsection{Calculation for the Number of Pulse Overlap}

Let $n_{1}, n_{2}, n_{3} \in\{0,1,2 \ldots\} . n_{1}$ is the modulus from dividing $\tau_{1}$ by $g, n_{2}$ is the modulus from dividing $L_{2}$ by $g$, and $n_{3}$ is the modulus from dividing $W_{2}$ by $g$, and define $0 \leq \delta_{1}, \delta_{2}, \delta_{3}<g$ as the remainders from dividing $\tau_{1}$ and $L_{2}$ and $W_{2}$ by $g$, respectively. We can put:

$$
\left\{\begin{array}{l}
\tau_{1}=n_{1} \cdot g+\delta_{1} \\
L_{2}=n_{2} \cdot g+\delta_{2} \\
W_{2}=n_{3} \cdot g+\delta_{3}
\end{array}\right.
$$

The $k$-th element of $S_{r}\left(\Delta t_{0}\right)$ in Equation (16) is $\varepsilon_{0}+(k-1) \cdot g$. If $L_{2}$ overlaps $\mathrm{PL}_{1}$ fully, then by Equation (3), $0<\varepsilon_{0}+(k-1) \cdot g-W_{2}<L_{2}-\tau_{1}$ must be satisfied. Combining Equation (17), we obtain:

$$
n_{3}+1+\frac{\delta_{3}-\varepsilon_{02}}{g}<k<n_{2}-n_{1}+n_{3}+1+\frac{\delta_{2}-\delta_{1}+\delta_{3}-\varepsilon_{0}}{g}
$$

Subject to $\left|\delta_{3}-\varepsilon_{0}\right|<g$, the minimum value of $k$ represented by $k_{\min }$ is expressed as follows:

$$
k_{\min }=\left\{\begin{array}{lll}
n_{3}+2 & \text { if } 0 \leq \varepsilon_{0} \leq \delta_{3} \\
n_{3}+1 & \text { if } \quad \delta_{3}<\varepsilon_{0}<g
\end{array}\right.
$$

Subject to $-g<\delta_{2}-\delta_{1}+\delta_{3}<2 g$ and $2 g>\delta_{2}-\delta_{1}+\delta_{3}-\varepsilon_{0}>-2 g$, the maximum value of $k$ represented by $k_{\max }$ can be expressed as follows:

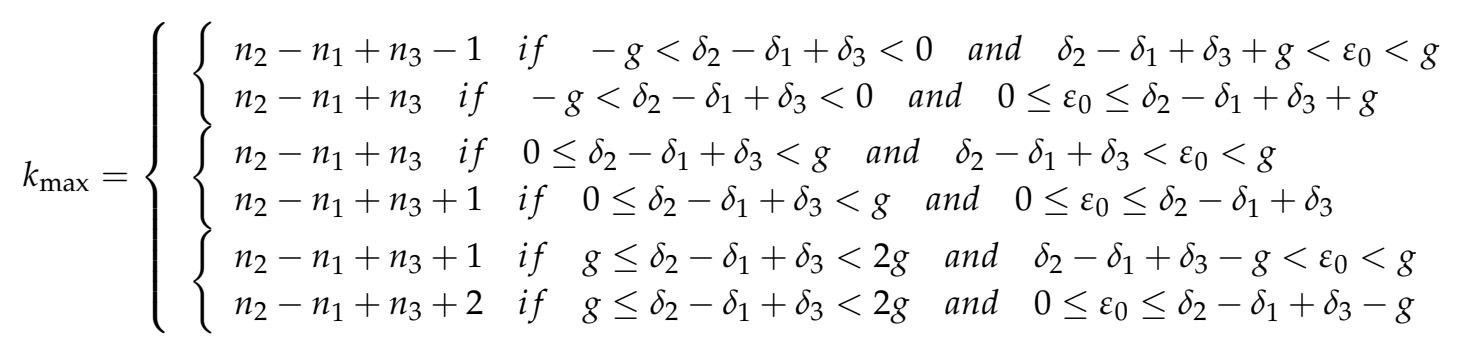

So the number $N_{\text {all_ov }}$ of $L_{2}$ overlapping with $\mathrm{PL}_{1}$ pulse fully in one overlap cycle is calculated to be:

$$
N_{\text {all_ov }}=k_{\max }-k_{\min }+1
$$

Let $k_{\text {max } \_ \text {max }}$ denote the maximum value of $k_{\max }, k_{\min \_\max }$ denote the maximum numerical value of $k_{\min }$, and $\delta_{d}$ denote the cutoff value of $\varepsilon_{0}$. For example, if $\leq \delta_{2}-\delta_{1}+\delta_{3}<g$, then 
$k_{\text {max } \_ \text {max }}=n_{2}-n_{1}+n_{3}+1$ and $\left.\delta_{d}=\delta_{2}-\delta_{1}+\delta_{3}\right]$; if $0 \leq \varepsilon_{0} \leq \delta_{3}$, then $k_{\text {min_max }}=n_{3}+2$. The variation of $k_{\text {max } \_ \text {max }}$ and $k_{\text {min_max }}$ with respect to $\varepsilon_{0}$ is shown in Figure 5 .

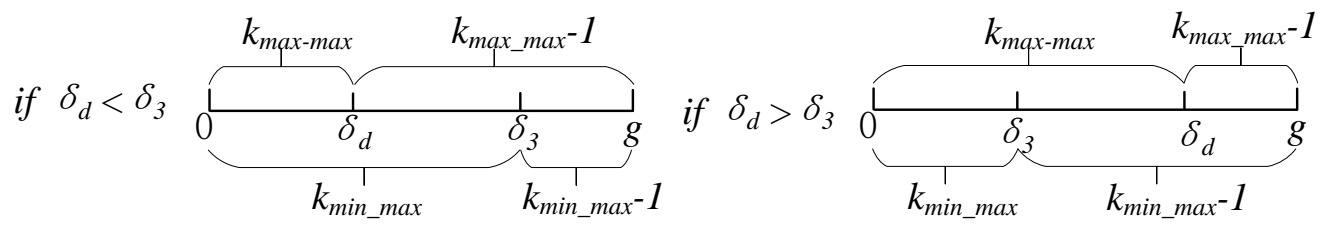

Figure 5. The variation of $k_{\text {max_max }}$ and $k_{\text {min_max }}$ with respect to $\varepsilon_{0}$.

Now we consider the calculation for $N_{a l l \_o v}$, for any $\varepsilon_{0}, N_{\text {all_ov }}$ can be calculated as follows:

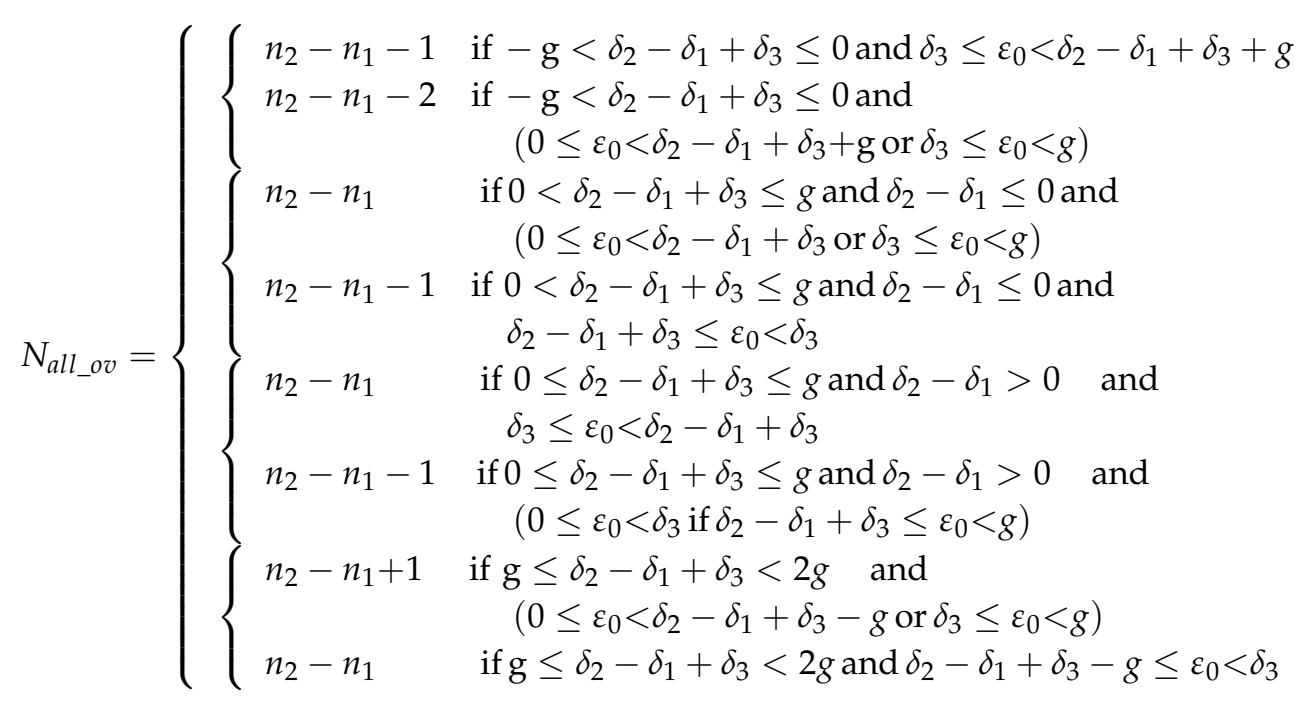

So $N_{\text {all_ov }}$ is determined by $n_{1}, n_{2}, \delta_{1}, \delta_{2}, \delta_{3}, g$ and $\varepsilon_{0}$. If $-g<\delta_{2}-\delta_{1}+\delta_{3}<0, N_{\text {all_ov }}$ changes with $\varepsilon_{0}$, the mean value of $N_{a l l \_o v}$ in a period of $\varepsilon_{0}$ represented by $N_{a l l \_o v \_a v}$ can be calculated as follows:

$$
\begin{aligned}
N_{a l l \_o v \_a v} & =\frac{\left(n_{2}-n_{1}-2\right) \cdot\left(2 g+\delta_{2}-\delta_{1}\right)}{g}+\frac{\left(n_{2}-n_{1}-1\right) \cdot\left(\delta_{2}-\delta_{1}+g\right)}{g} \\
& =\frac{\delta_{2}-\delta_{1}+\left(n_{2}-n_{1}\right) \cdot g}{g}=\frac{L_{2}-\tau_{1}}{g}
\end{aligned}
$$

Similarly, if $0<\delta_{2}-\delta_{1}+\delta_{3} \leq g$ or $g<\delta_{2}-\delta_{1}+\delta_{3} \leq 2 g$, the expression of $N_{\text {all_ov } a v}$ is the same as Equation (23). If $T_{1} \bmod T_{2}=0$, according to Equation (8), all the elements of $S_{r}\left(\Delta t_{0}\right)$ is equal to $\Delta t_{0} \bmod T_{2}$ and the period of $\Delta t_{0}$ is $\left.T_{2}\right]$, so $N_{\text {all_ov }}$ can be expressed as follows:

$$
N_{\text {all_ov }}=\left\{\begin{array}{lll}
0 & \text { if } & 0<\Delta t_{0}-W_{2} \leq L_{2}-\tau_{1} \\
\frac{T_{2}}{g} & \text { if } & \Delta t_{0}-W_{2}>L_{2}-\tau_{1}
\end{array}\right.
$$

So $N_{\text {all_ov_av }}$ can be calculated as follows:

$$
N_{a l l \_o v \_a v}=\frac{\left(L_{2}-\tau_{1}\right) \cdot \frac{T_{2}}{g}+\left(T_{2}-W_{2}-L_{2}+\tau_{1}\right) \cdot 0}{T_{2}}=\frac{L_{2}-\tau_{1}}{g}
$$

Clearly, Equation (25) is the same as Equation (23), so $N_{a l l \_o v \_a v}$ is determined by $L_{2}, \tau_{1}$ and $g$ whether $T_{1}$ mod $T_{2}=0$ or not. Similarly, the mean value of $\mathrm{PL}_{1}$ pulses overlapped fully by $S_{2}$ is $\frac{S_{2}-\tau_{1}}{g}$. 


\subsection{Calculation for the Probability of Pulse Overlap}

The overlap probability in one cycle represented by $P_{\text {all_ov }}$ can be defined as follows:

$$
P_{a l l \_v}=\frac{N_{a l l \_o v}}{N_{T_{1}}}
$$

Combining Equations (6) and (22), the variation of $N_{\text {all_ov }}$ is 1 in one overlap cycle, so the variation of $P_{a l l \_o v}$ represented by $\Delta P_{\text {all_ov }}$ can be calculated as follows:

$$
\Delta P_{\text {all_ov }}=\frac{\Delta N_{\text {all_ov }}}{N_{T_{1}}}=\frac{g}{T_{2}}
$$

Let $f_{1}$ and $f_{2}$ denote the PRF of ATCRBS and DME, respectively, and suppose $f_{2}=\frac{a}{b} \cdot f_{1}$ ( $a$ and $b$ are natural and coprime numbers). So the great common divisor $g$ of $T_{1}$ and $T_{2}$ is calculated to be:

$$
g=\left(T_{1}, T_{2}\right)=\left(\frac{1}{f_{1}}, \frac{b}{a \cdot f_{1}}\right)=\frac{1}{a \cdot f_{1}}
$$

Hence:

$$
\Delta P_{\text {all } \_ \text {ov }}=\frac{g}{T_{2}}=\frac{\frac{1}{a \cdot f_{1}}}{\frac{b}{a \cdot f_{1}}}=\frac{1}{b}
$$

Combining Equations (6), (23), and (26), the mean value of $P_{a l l \_o v}$ denoted by $P_{a l l \_o v \_a v}$ can be calculated as follows:

$$
P_{a l l \_a v \_a v}=\left\{\begin{array}{lll}
\frac{\left(\frac{L_{2}-\tau_{1}}{g}+\frac{S_{2}-\tau_{1}}{g}\right) \cdot g}{T_{2}}=1-\frac{2 W_{2}+2 \tau_{1}}{T_{2}} & \text { if } \quad S_{2}>\tau_{1} \text { and } L_{2}>\tau_{1} \\
\frac{S_{2}-\tau_{1}}{g \cdot \frac{T_{2}}{g}}=1-\frac{2 W_{2}+L_{2}+\tau_{1}}{T_{2}} & \text { if } \quad S_{2}>\tau_{1} \text { and } L_{2} \leq \tau_{1}
\end{array}\right.
$$

\section{Results and Discussion}

To verify the validity of PPOM, we calculate and simulate the impact of DME interference on ATCRBS using PPOM, PDM, and MCM, respectively, and then study the results based on different methods. Moreover, we analyze the impact of PRF and difference of initial time on recognition probability. In this paper, we focus on the interference impact of DME interference on ATCRBS in the time domain, some assumptions are made as follows:

- Probability of recognition equals that of non-overlapping in the time domain

- Signal propagation environment characteristics are neglected

- Time of signal processing in the equipment is negligible

- $\quad$ Aircraft is motionless

- Interference comes from the DME only

- Interrogation antenna is not rotational.

\subsection{Calculation and Simulation Based on Different Methods}

\subsubsection{Calculation Based on PPOM}

One-path DME interference is assumed to be coexisted with ATCRBS signal. Using Equation (30), Mean Recognition Probability (MRP) for ATCRBS interfered by DME is calculated as in Table 1. The interference name is shown in the first row of Table 1, DMEInterX and DMERepX denote interrogation and reply signal operating in mode $X$, respectively, and DMEY denotes interrogation or reply signal 
operating in mode Y. ATCRBS signal is shown in the first column of Table 1, SSR A and SSR C denote the interrogation signal operating in mode A and mode $C$, respectively. ATC denotes the reply signal without SPI and ATC_SPI denotes the reply signal with SPI. MRP is shown in the cell intersected by the row and column correspondingly, where $f_{2}$ (in MHz) is PRF of DME signal.

For example, when interfering signal is DMEInterX and wangted signal is SSR A, From Figure 2 duration of wanted signal is $\tau_{1}=8.8 \mu \mathrm{s}$, from Figure 1 we can derive that the intra idle time of DME pulse pair $L_{2}=I_{2}-W_{2}=12-3.5=8.5 \mu$ s and the inter idle time DME pulse pair $S_{2}=T_{2}-I_{2}-W_{2}=\frac{1}{f_{2}}-15.5$, considering that PRF of DME signal $f_{2}<2800 \mathrm{~Hz}$ [12], i.e., $T_{2}>357 \mu \mathrm{s}$, hence $S_{2}>\tau_{1}$. Therefore, MRP can be calculated as Equation (30), combining that $L_{2}<\tau_{1}$, we get:

$$
P_{a l l \_o v \_a v}=1-\left(2 W_{2}+L_{2}+\tau_{1}\right) \cdot f_{2}=1-(2 \times 3.5+8.5+8.8) \cdot f_{2}=1-24.3 \cdot f_{2} .
$$

Similarity, the other MRP in Table 1 can be calculated as mentioned above.

Table 1. Calculation for the mean recognition probability.

\begin{tabular}{cccc}
\hline & $\begin{array}{c}\text { DMEInterX } \\
\left(\boldsymbol{L}_{2}=8.5 \boldsymbol{\mu s}\right)\end{array}$ & $\begin{array}{c}\text { DMERepX } \\
\left(\boldsymbol{L}_{\mathbf{2}}=\mathbf{2 6 . 5} \boldsymbol{\mu \mathrm { s }}\right)\end{array}$ & $\begin{array}{c}\text { DMEY } \\
\left(\boldsymbol{L}_{2}=\mathbf{3 2 . 5} \boldsymbol{\mu s}\right)\end{array}$ \\
\hline SSR A $\left(\tau_{1}=8.8 \mu \mathrm{s}\right)$ & $1-24.3 \cdot f_{2}$ & $1-24.6 \cdot f_{2}$ & $1-24.6 \cdot f_{2}$ \\
SSR C $\left(\tau_{1}=21.8 \mu \mathrm{s}\right)$ & $1-37.3 \cdot f_{2}$ & $1-50.6 \cdot f_{2}$ & $1-50.6 \cdot f_{2}$ \\
ATC $\left(\tau_{1}=20.75 \mu \mathrm{s}\right)$ & $1-33.1 \cdot f_{2}$ & $1-48.5 \cdot f_{2}$ & $1-48.5 \cdot f_{2}$ \\
ATC_SPI $\left(\tau_{1}=25.15 \mu \mathrm{s}\right)$ & $1-40.65 \cdot f_{2}$ & $1-57.3 \cdot f_{2}$ & $1-57.3 \cdot f_{2}$ \\
\hline
\end{tabular}

Table 1 shows that MRP is a linear decreasing function of $f_{2}$ when the operation mode of DME and ATCRBS are selected. Moreover, the calculation formula of MRP for DME RepX interference on ATCRBS is the same as that for DME Y interference, because $L_{2}$ in DME RepX or DME $Y$ is larger than the ATCRBS signal duration. From Table 1, it can be seen that the operation mode of DME or ATCRBS has effect on $R_{e}$. For example, when interference is DME Y and its $\operatorname{PRF} f_{2}$ is equal to the maximum value $2700 \mathrm{~Hz}$, the reply signal is ATC_SPI, then $R_{e}$ can be calculated as $\left(1-50.6 f_{2}\right) \cdot\left(1-57.3 f_{2}\right)=0.7298$ when ATCRBS is operating in mode $\mathrm{C}$, while $R_{e}$ is calculated by $\left(1-24.6 f_{2}\right) \cdot\left(1-48.5 f_{2}\right)=0.8113$ when ATCRBS is operating in mode A without SPI. Similarity, when interference is DME InterX and its $\operatorname{PRF} f_{2}$ is equal to the minimum value $10 \mathrm{~Hz}$, the reply signal is ATC, $R_{e}$ can be calculated as $\left(1-24.3 f_{2}\right) \cdot\left(1-33.1 f_{2}\right)=0.9994$ when ATCRBS is operating in mode A.

\subsubsection{Calculation for MRP Based on PDM}

The coexistence of $n(n \geq 1)$ paths DME signal and one-path ATCRBS signal is assumed to constitute a pulse-stream. Since DME signal is composed of two pulses in one cycle, the pulse density of the pulse-stream can be defined as follows:

$$
\lambda=\lambda_{i}+\lambda_{s}=2 \cdot \sum_{j=1}^{n} f_{j}+f_{1}
$$

where $\lambda$ is the total pulse density of pulse-stream, $\lambda_{i}$ is the pulse density of DME pulse-stream, $\lambda_{s}=f_{1}$ is the pulse density of ATCRBS pulse, $f_{1}$ is PRF of ATCRBS signal, and $f_{j}$ is PRF of the $j-t$ th path DME signal.

If $n$ is sufficiently large, the distribution of the arrival time from pulse-stream to ATCRBS can be considered to be in accordance with the Poisson distribution. There are two pulses next to an ATCRBS signal in the pulse-stream; one is in front of the signal, the other is behind the signal. ATCRBS signal is interfered if one of the pulses next to it overlaps with it. The front pulse in the pulse-stream cannot overlap 
with ATCRBS signal if the pulse is also an ATCRBS signal, because the duty cycle of an ATCRBS signal is larger than 1. $\frac{\lambda_{s}}{\lambda}$ and $\frac{\lambda_{i}}{\lambda}$ are the probabilities that the front pulse is ATCRBS signal and DME signal, respectively. The probability of DME signal non-overlapped by the back pulse in the pulse-stream is $e^{-\lambda \cdot W_{2}}$. Similarly, $e^{-\lambda \cdot \tau_{1}}$ is the probability of ATCRBS non-overlapped by the back pulse in the pulse-stream. Therefore, the probability of ATCRBS signal duration non-overlapped by a DME pulse in the pulse-stream $P_{d}$ can be calculated as follows:

$$
P_{d}=\left(e^{-\lambda \cdot W_{2}} \cdot \frac{\lambda_{i}}{\lambda}+\frac{\lambda_{s}}{\lambda}\right) \cdot e^{-\lambda \cdot \tau_{1}}
$$

\subsubsection{MRP and MRN vs PRF Based on Different Methods}

The interference is one-path DME interrogation signal operating in mode $X$, the PRF of interference is $1000 \sim 2800 \mathrm{~Hz}$ with $200 \mathrm{~Hz}$ interval. The desired signal is ATCRBS reply with SPI with $25.15 \mu$ s pulse duration and $2000 \mathrm{~Hz}$ PRF. The simulation times of MCM are 8000, and the initial time of DME and ATCRBS are the uniform distribution of data among their respective periods. Using PPOM, the MRP of ATCRBS signal is calculated by $P_{\text {all_ov_av }}=1-40.65 \cdot f_{2}$ shown in Table 1 , and the mean recognition number (MRN) of ATCRBS can be calculated as Equation (23). The results based on different methods are depicted in Figure 6. MRP is plotted on the left $Y$ axis, MRN is plotted on the right $Y$ axis, and the $X$ axis represents the PRF of DME.

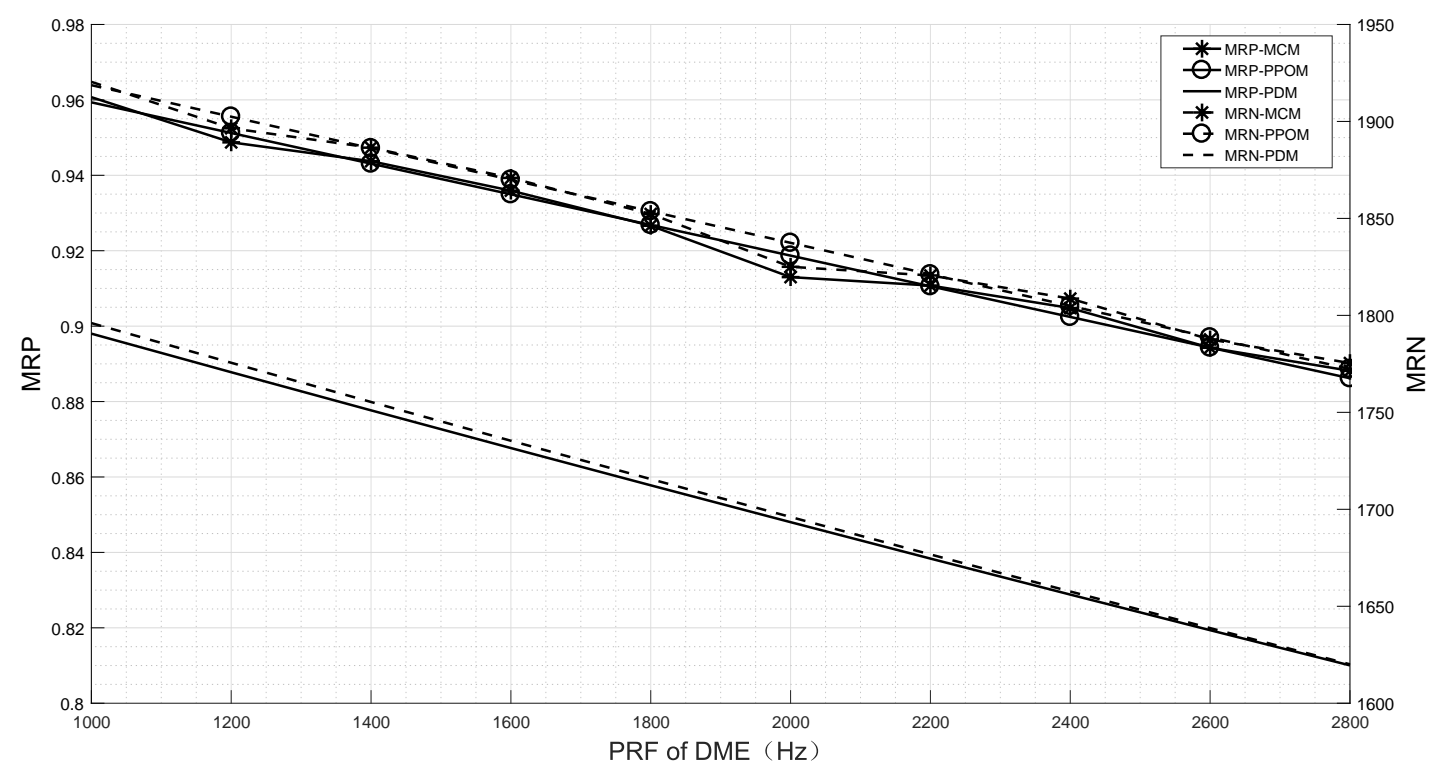

Figure 6. Mean Recognition Probability (MRP) and Mean Recognition Number (MRN) based on different methods.

Figure 6 shows that MRP based on PPOM agrees well with that based on MCM, while MRP based on PDM is less than MRP based on MCM, the reason is that the pulse-stream composed of one channel DME signal and ATCRBS signal does not in agreement with Poisson distribution at all. PDM cannot be used to calculate MRP if the number of DME interference source is a small amount. However, if the number of DME interference source is large enough and the interference sources are statistical independence, furthermore, the PRF of DME is the uniform distribution of data from $30 \mathrm{~Hz}$ to $150 \mathrm{~Hz}$; consequently, this kind of pulse-stream can be considered to accord with Poisson distribution. 
The total MRP of ATCRBS signal is equal to the product of MRP non-overlapped by the entire DME signal. The total MRP with respect to the paths of DME signal is depicted in Figure 7. The number of interference source is (1 626) with 25 interval. The number on the curve is pulse-stream density (in pulses per second), for instance, when the number of interference source is 101, the pulse-stream density is 20,518 pulses per second.

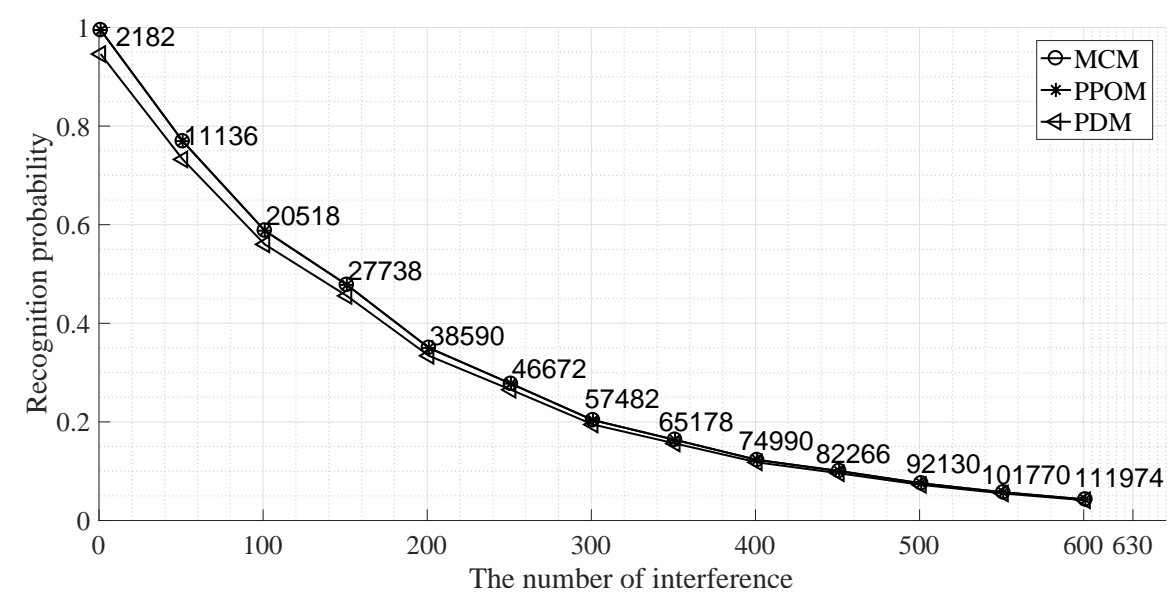

Figure 7. MRP vs the number of interference source.

Figure 7 shows that the result based on MCM is almost the same as that based on PPOM, just as shown in Figure 6. In addition, the difference between MRP based on PDM and MRP based on PPOM gets smaller and smaller as the number of interference sources increases. In other words, MCM can be substituted by PDM to calculate MRP when the number of DME interference sources is large enough.

\subsection{Variation of Recognition Probability vs Multiples of PRF}

As mentioned above, the variation of overlap probability between two periodic pulse signals is periodic. Moreover, according to Equation (29), the maximum variation of recognition probability $\Delta P_{a l l \_o v}$ is equal to $\frac{1}{b}$ when initial time difference varies in a period, which means that $\Delta P_{\text {all_ov }}$ can be adjusted through changing the multiples between DME PRF $\left(f_{2}\right)$ and $\operatorname{ATCRBS} \operatorname{PRF}\left(f_{1}\right)$. If the interfering signal is DME RepX, the desired signal is ATC and $f_{1}$ is $1996 \mathrm{~Hz}$. When $f_{2}$ initiates from $700 \mathrm{~Hz}$ to $2700 \mathrm{~Hz}$ with $0.1 \mathrm{~Hz}$ interval, the variation of $\Delta P_{\text {all_ov }}$ is depicted in Figure 8 . It can be seen that $\Delta P_{\text {all_ov }}$ ranges from 0 to 1 . Moreover, when $f_{2}$ is close to the integer multiple of $f_{1}, \Delta P_{\text {all_ov }}$ is close to zero, and the closer to the integer multiple, the closer to zero. However, once $f_{2}$ is an integer multiple of $f_{1}$, the difference value equals to 1 . 


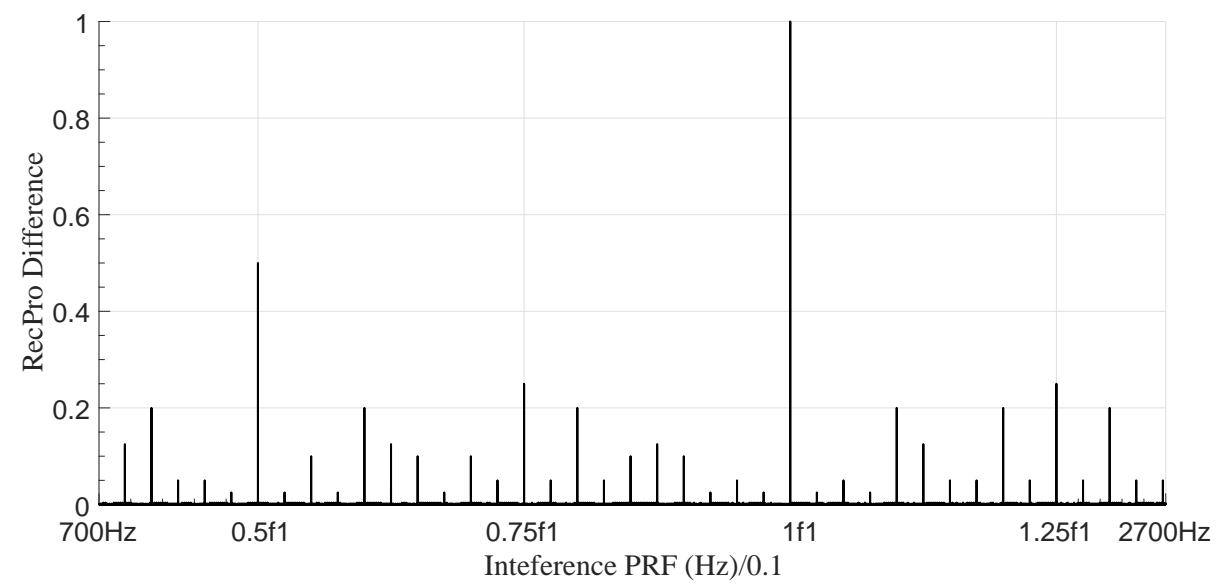

Figure 8. The maximum variation of recognition probability vs Pulse Recurrence Frequency (PRF).

\subsection{Analysis of Recognition Probability Varying with Difference of Initial Time}

According to Equation (22), the recognition probability varies with difference of initial time. If the interference is DME RepX and its PRF $f_{2}$ is $2561 \mathrm{~Hz}$, the desired signal is SSR C and its PRF $f_{1}$ is $394 \mathrm{~Hz}$. Figure 9 illustrates the recognition probability varying with the difference of initial time in a cycle. The definitions of $\delta_{d-1}$ and $\delta_{d-2}$ are the same as $\delta_{d}$ shown in Figure 5, and the definitions of $\delta_{3-1}$ and $\delta_{3-2}$ are the same as $\delta_{3}$ shown in Figure 5, and $\delta_{3-1}=W_{2} \bmod g, \delta_{3-2}=\left(L_{2}+2 W_{2}\right) \bmod g$. There are two idle durations in DME RepX pulse signal whose durations are all larger than that of ATCRBS signal, so there are four jumping points at $\delta_{3-1}, \delta_{3-2}$, and $\delta_{3-3}$. From Figure 9 , it can be seen that the MRP is 0.87041 according to the formula $1-50.6 \cdot f_{2}$ shown in Table 1 , the maximum value of recognition probability difference is 0.5 according to Equation (29). It is indicated that the distribution of recognition probability with respect to difference of initial time can be adjusted through changing ATCRBS PRF under the condition of keeping the MRP unchanged.

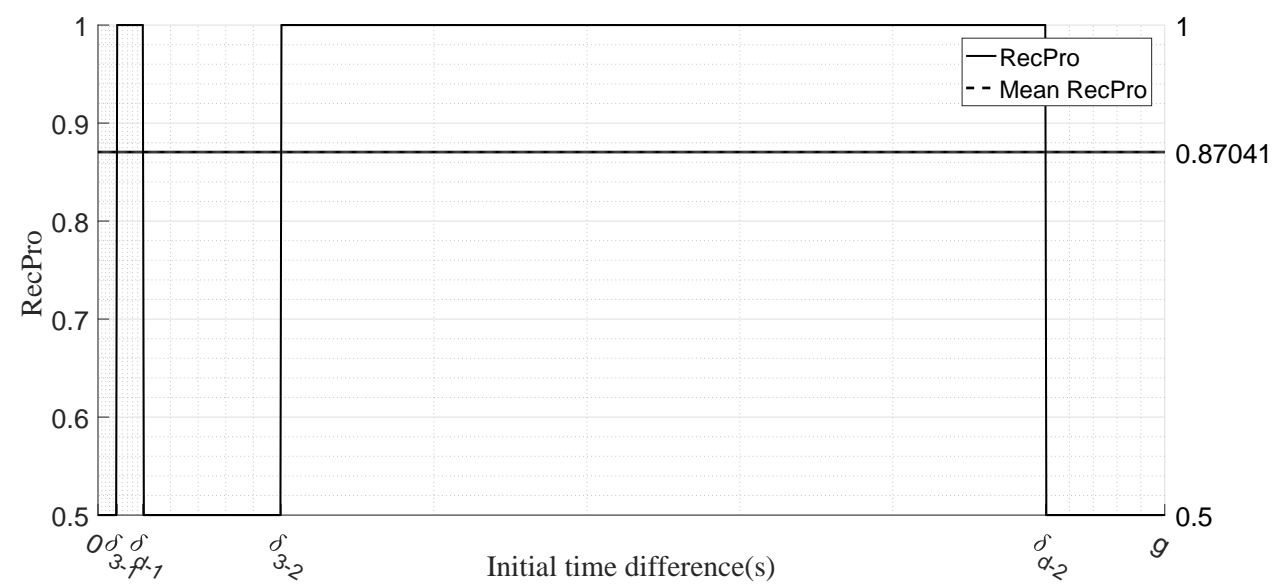

Figure 9. The recognition probability vs initial time.

\section{Conclusions}

From the above discussion, the conclusion can be reached that PPOM is an accurate and efficient method to analyze interference between two or more periodic pulse signals. Moreover, PPOM can not only 
be used to analyze EMC, but it can also be used to reduce or avoid interference from the enemy equipment in electronic warfare. Equally important is that using PPOM, we can reduce or avoid interference by adjusting PRF or difference of initial time. Moreover, the maximum difference value and distribution of overlap probability in one overlap cycle can also be changed.

The interference from DME to ATCRBS in the time domain is analyzed based on PPOM, and the results show that the mean reply efficiency ranges from 0.7298 to 0.9994 , as calculated in Section 4.1.1. It is worth noting that the mean reply efficiency can be adjusted by changing operation mode of DME or ATCRBS, for example, other things being equal, the reply efficiency interfered by DME operating in mode $\mathrm{X}$ is larger than that interfered by DME operating in mode $\mathrm{Y}$, and the reply efficiency operating in mode $\mathrm{A}$ is larger than that operating in mode $C$.

The limitation of PPOM is that it is derived from the periodicity of the signal; the statistical value of overlap probability can be obtained only if the signal is not periodic. Moreover, in this paper, the time of signal processing in the equipment is neglected, in fact, after recognition of a proper signal, the equipment shall not reply to any other signals for a long time, referred to as dead time. For example, the dead time of a transponder shall end no later than 125 microseconds after the transmission of the last reply pulse of the group [12], so the recognition probability in this paper is larger than the actual value. Furthermore, we did not take into account signal propagation environment characteristics. As a further step, to corroborate with the actual condition, some assumptions should be taken into account.

Author Contributions: G.J. and H.Y. designed and performed the research. G.J. and Y.F. wrote the manuscript. G.J. and Y.F. performed the computation. G.J., P.Y. and Y.F. analyzed the data. All authors discussed the results and commented on the manuscript.

Funding: This research received no external funding.

Conflicts of Interest: The authors declare no conflict of interest.

Abbreviations
The following abbreviations are used in this manuscript
$\begin{array}{ll}\text { DME } & \text { Distance Measuring Equipment } \\ \text { ATCRBS } & \text { Air Traffic Control Radar Beacon System } \\ \text { SSR } & \text { Secondary Surveillance Radar } \\ \text { ATC } & \text { Air Traffic Control } \\ \text { EMC } & \text { Electromagnetic Compatibility } \\ \text { PPOM } & \text { Periodic Pulse Overlap Method } \\ \text { DME } & \text { Distance Measuring Equipment } \\ \text { MCM } & \text { Monte Carlo Method } \\ \text { PDM } & \text { Poisson Distribution Method } \\ \text { MRP } & \text { Mean Recognition Probability } \\ \text { MRN } & \text { Mean Recognition Number }\end{array}$

\section{References}

1. Krishnan, N.N.; Kumbhkar, R.; Mandayam, N.B.; Seskar, I.; Kompella, S. How Close Can I Be?-A Comprehensive Analysis of Cellular Interference on ATC Radar. In Proceedings of the 2017 IEEE Global Communications Conference, Singapore, 4-8 December 2017; pp. 1-6.

2. Onrubia, R.; Querol, J.; Pascual, D.; Alonso-Arroyo, A.; Park, H.; Camps, A. DME TACAN Impact Analysis on GNSS Reflectometry. IEEE J. Sel. Top. Appl. Earth Obs. Remote Sens. 2016, 9, 4611-4620. [CrossRef]

3. Houdzoumis, V.A. A Simplified Method for the Analysis of Interference from JTIDS Radio Networks to DME Aeronautical Radionavigation Systems. J. Navig. 2009, 62, 721-737. [CrossRef] 
4. Otsuyama, T.; Ozeki, S. A study of evaluation method for aeronautical L-band signal environment during flight experiments. In Proceedings of the 2014 International Symposium on Electromagnetic Compatibility, Gothenburg, Sweden, 1-4 September 2014; pp. 781-784.

5. Najett, N.; Lacerda, R.D.; Azoulay, A.; Letertre, T. Electromagnetic Compatibility from a Time-Frequency Perspective. Rev. Telecomun. 2012, 13, 60-69.

6. Saaifan, K.A.; Elshahed, A.; Henkel, W. Cancellation of Distance Measuring Equipment Interference for Aeronautical Communications. IEEE Trans. Aerosp. Electron. Syst. 2017, 53, 3104-3114. [CrossRef]

7. Bellido-Manganell, M.A. Impact assessment of LDACS on JTIDS. In Proceedings of the 2017 Integrated Communications, Navigation and Surveillance Conference (ICNS), Herndon, VA, USA, 8-20 April 2017.

8. Najett, N.; Raul De, L.; Alain, A.; Thierry, L. Radio Systems Coexistence from a Time Domain Perspective: principle and example. In Proceedings of the International Workshop on Telecommunications (IWT), Rio de Janeiro, Brazil, May 2011.

9. Najett, N.; De Lacerda, R.; Azoulay, A.; Letertre, T.; Outtier, O. Cosite Coexistence between Future L-DACS2 and Legacy DME Systems. IEEE Trans. Aerosp. Electron. Syst. 2013, 49, 2585-2600.

10. Hoki, B.; Jaesung, L. Spectrum Sharing for Coexistence of Fixed Satellite Services and Frequency Hopping Tactical Data Link. IEEE J. Sel. Areas Commun. 2016, 34, 2642-2649, [CrossRef]

11. Lo, S.C.; Enge, P. Assessing the Capability of Distance Measuring Equipment to Support Future Air Traffic Capacity. J. Inst. Navig. 2012, 59, 249-261. [CrossRef]

12. ICAO. Aeronautical Telecommunications, 6th ed.; ICAO: Montreal, OC, Canada, 2006.

13. Guo, J.; Zhang, W.; Ye, J.; Fan, X.; Pan, J.; Du, H.; Zhang, Y. Technical Standards for ATC Secondary Surveillance Radar; Civil Aviation Administration of China: Beijing, China, 2017.

14. Pan, C.; Pan, C. Elementary Number Theory, 3rd ed.; Peking University Press: Beijing, China, 2013.

(C) 2019 by the authors. Licensee MDPI, Basel, Switzerland. This article is an open access article distributed under the terms and conditions of the Creative Commons Attribution (CC BY) license (http://creativecommons.org/licenses/by/4.0/). 